\title{
Optical Properties of CuS Nanoparticles Embedded Polyvinyl Alcohol (PVA) Films
}

\author{
L.S. Chongad ${ }^{1,2}$, Amit Jain²,3, G.S. Mukherjee ${ }^{4}$ M. Banerjee2,** \\ 1 Department of Physics, Government Arts and Science College, Ratlam, 457001 Madhya Pradesh, India \\ 2 Nanoscience and Nanotechnology Laboratory, School of Physics, Devi Ahilya University, Khandwa Road, Indore, \\ 452001 Madhya Pradesh, India \\ ${ }^{3}$ Department of Engineering Sciences and Humanities, Indore Institute of Science and Technology, Pithampur \\ Road, Rau, 453331 Indore Madhya Pradesh, India \\ ${ }^{4}$ Defence Research \& Development Organization (DRDO), Metcalfe House DRDO Complex, 110054 Delhi, India
}

(Received 08 January 2021; revised manuscript received 10 February 2021; published online 25 February 2021)

\begin{abstract}
The pristine poly(vinyl alcohol) and CuS nanoparticles with different concentrations (1\%-10\%) doped PVA films were prepared by solution casting method. The CuS nanoparticles were prepared by chemical precipitation; and XRD studies confirmed the formation of pure CuS nanoparticles with average size of $18 \mathrm{~nm}$. The optical properties of pristine as well as doped PVA films were evaluated using UV-VIS-NIR spectroscopy. UV-VIS-NIR spectrum of CuS nanoparticles showed LSPR band in NIR region. The optical properties like absorption coefficient, bandgaps, high frequency refractive index, and metallization criterion have been evaluated; both the direct and indirect band gaps decreased in case of doped PVA films; and the results and the features are discussed.
\end{abstract}

Keywords: PVA, CuS nanoparticles, Optical properties, Refractive index, Metallization.

DOI: $10.21272 /$ jnep.13(1).01023

PACS numbers: 36.20.Kd, 78.67.Sc, 78.20.Ci,

85.40.Ls

\section{INTRODUCTION}

In recent years optical properties of polymers are drawing great interest because of their scope for application in optical devices. The versatility and dependability of polymers is established from the fact that they have attractive properties like lightweight, good mechanical and optical properties [1]. Polymers are also considered to be good host materials where dopant can modify the physicochemical properties [1-3]. The optical band gap and refractive index are the fundamental parameters of an optical material, because these are directly linked to the electronic properties of the material [4]. High refractive index of optical materials is needed in various fields including optical waveguides, LED encapsulation materials, etc. [5]. Poly (vinyl alcohol) (PVA) is a polymer which is eco-friendly, water soluble and has good film forming capability; this also acts as a good host. PVA has been doped with dopant such as polymers, metal salts, and ions to obtain desired properties [6-7]. Many papers reported studies on structural and optical properties of additive doped PVA systems [8-13]. It is reported to be a nice matrix material to accept metal nanoparticles as has been observed that Co nanoparticles can be embedded in the matrix of PVA to display magnetic properties [8, 14]; and with gold nanoparticles captured in the PVA matrix system can provide characteristic optical properties [15]. In this paper we report optical studies on PVA films doped with $\mathrm{CuS}$ nanoparticles. The prepared films were characterized by UV-VIS spectroscopy and various optical properties like absorption coefficient, band gap, refractive index and metallization parameter have been evaluated and the results are reported here.

\section{EXPERIMENTAL DETAIL}

\subsection{Sample Preparation}

CuS nanoparticles were prepared by chemical precipitation method described earlier [16]. In the present work, starting material was copper sulphate, purchased from Aldrich and was used as received. An N/20 aqueous solution of copper sulphate was prepared and CuS nanoparticles were obtained by chemical precipitation method [16] by maintaining $\mathrm{pH}$ value of 8 . The CuS nanoparticles thus prepared were incorporated in PVA film. PVA (Mol; wt. $72 \mathrm{kDa}$ ) used was from Merck, (Germany) Pristine PVA films as well as $1 \%, 3 \%, 5 \%$ and $10 \%$ CuS nanoparticles doped PVA films were prepared by solution casting method, the details are same as in Ref. [2]. The thickness of the films was ca. $60 \mu \mathrm{m}$.

\subsection{Physical Measurements}

The X-ray diffraction (XRD) analysis of the prepared sample of CuS nanoparticles was done using Burker D8 Advance X-ray diffractometer with $\mathrm{Cu}-\mathrm{K}-\alpha$ X-rays of wavelength $\lambda=1.54 \AA$ and operated at $40 \mathrm{kV}$ and $100 \mathrm{~mA}$. The data was taken for the $2 \theta$ range $10^{\circ}$ to $60^{\circ}$ with a step of $0.1792^{\circ}$. TEM image were recorded with Philips CM 200 microscope operated at $200 \mathrm{kV}$. UV-visible-NIR absorption spectra were recorded in the wavelength range of 190-1100 nm using a Shimadzu 2450 double beam spectrophotometer. All the measurements were made at room temperature.

\section{RESULTS AND DISCUSSION}

\subsection{XRD Studies of CuS Nanoparticles}

mandira_bm@rediffmail.com

The results were presented at the International Conference on Multifunctional Nanomaterials (ICMN2020) 
XRD patterns of the synthesized CuS NPs are shown in Fig. 1. The XRD diffraction peaks of nanoparticles (NPs) can be seen at $2 \theta=27.21^{\circ}, 27.38^{\circ}, 29.27^{\circ}$, $31.78^{\circ}, 32.85^{\circ}, 47.93^{\circ}, 52.71^{\circ}, 59.34^{\circ}$ corresponding to planes (100), (101), (102), (103), (006), 110), (108) and (116) of CuS nanoparticles which match well with the values of hexagonal phase reported in JCPDS Card 060464 indicating that $\mathrm{CuS}$ exists as covellite phase. Furthermore, no other peaks other than those corresponding to CuS are observed, which indicates the high purity of synthesized CuS NPs. The average crystallite size of CuS nanocrystallites as estimated by scherrer formula is found to be $18 \mathrm{~nm}$.

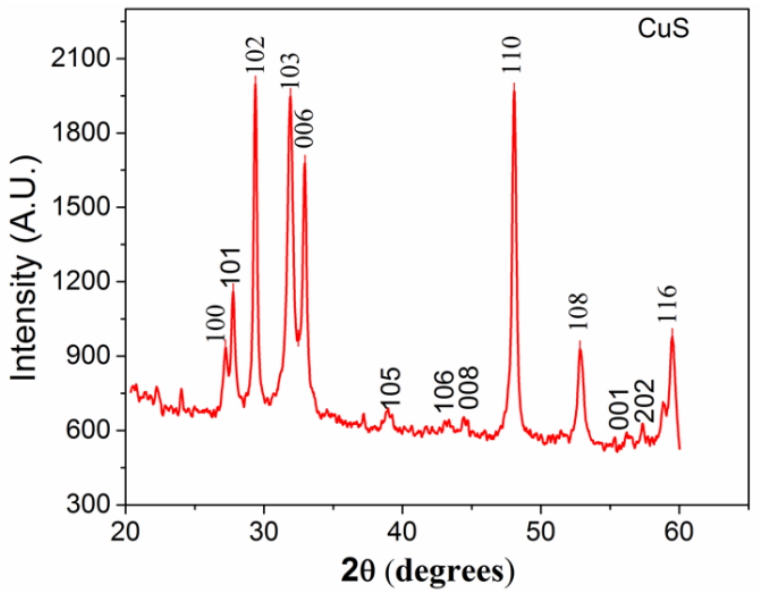

Fig. 1 - XRD patterns of the sample CuS Nanoparticles

\section{$3.2 \quad$ TEM Study}

TEM image of CuS nanoparticles recorded using is presented in Fig. 2; CuS nanoparticles exhibit disc like structure with hexagonal shape. The particle size as determined from TEM images is found to be $9-23 \mathrm{~nm}$.

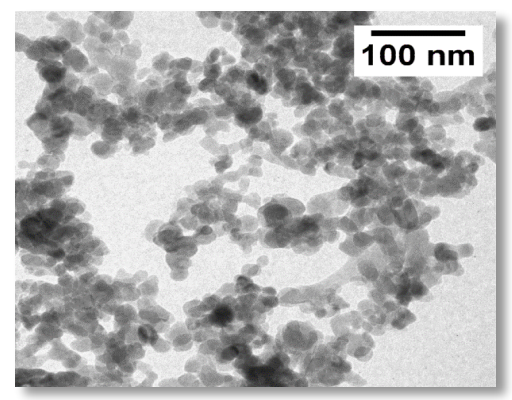

Fig. 2 - TEM image of CuS nanoparticles

\subsection{UV-visible Absorption Studies}

UV-Visible-NIR absorption spectroscopy is a useful tool for understanding the optical properties of the nanoparticles. Fig. 3. shows the UV-Vis-NIR absorption spectrum of the CuS nanoparticles, and a band around $837 \mathrm{~nm}$ extending into the NIR region can be observed, which can be attributed to localized surface Plasmon resonance (LSPR) band of CuS nanoparticles. The band gap of $\mathrm{CuS}$ nanoparticles is found to be $2.78 \mathrm{eV}$.

Absorption spectra of pure PVA and CuS nanoparticles doped PVA films are shown in Fig. 4. In case of pristine PVA, the absorption bands at around $280 \mathrm{~nm}$ and

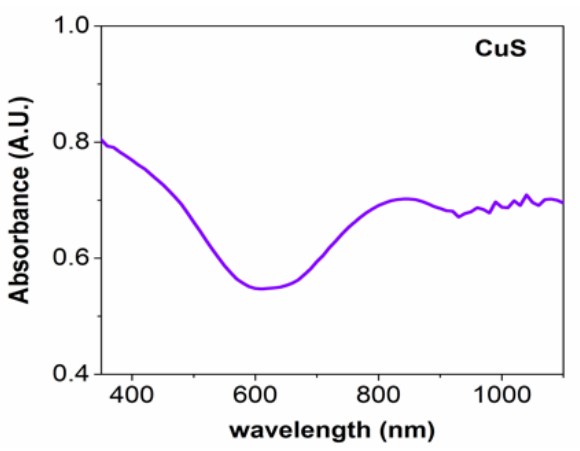

Fig. 3 - Absorption spectra of copper CuS nanoparticles

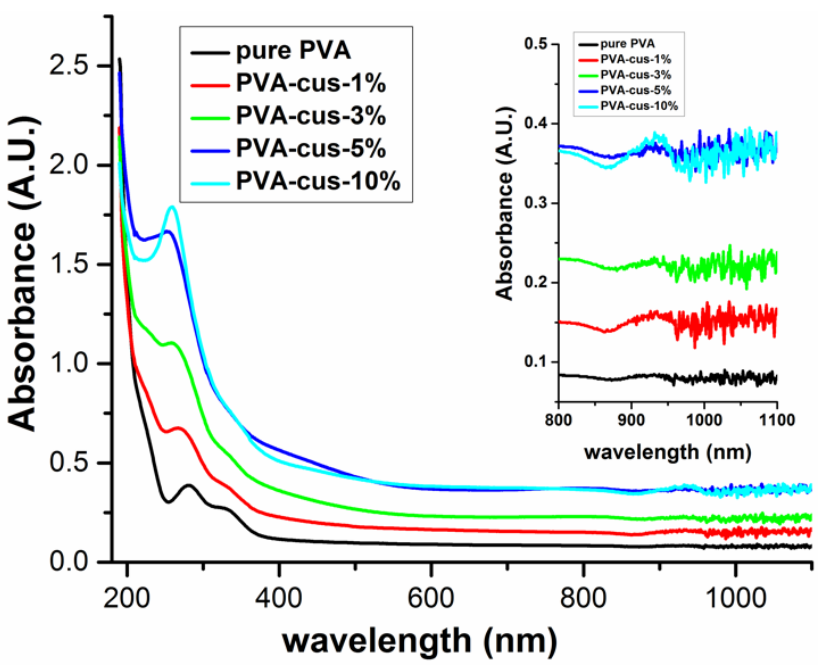

Fig. 4 - Plot of absorbance vs. wavelength of pure PVA and PVA-CuS films

$330 \mathrm{~nm}$ which can be assigned to transition of resonating $>\mathrm{C}=\mathrm{O}$ structures which can be attributed to $\pi-\pi^{*}$ and $n-\pi^{*}$ transition. From the absorption spectra of CuS doped PVA, it can be seen that the absorption band at $280 \mathrm{~nm}$ is shifted to $273 \mathrm{~nm}, 262 \mathrm{~nm}, 259 \mathrm{~nm}$, and 257 for PVA-CuS$1 \%$, PVA-CuS-3\%, PVA-CuS- $5 \%$ and PVA-CuS- $10 \%$ films respectively showing a blue shift of this band on incorporation of $\mathrm{CuS}$ nanoparticles, the shift gradually increasing with the increasing concentration of the dopant. The band at $330 \mathrm{~nm}$ is broadened in case of PVACuS-1 \% and PVA-Cus-3\% sample while for PVA-CuS$5 \%$ and PVA-CuS-10\% films absorption band at $330 \mathrm{~nm}$ is totally absent. Also the absorption bands are affected with CuS doping indicates the formation of complex chemical bonding of $\mathrm{CuS}$ nanoparticles with PVA.

Another interesting feature in the absorption spectra of this system is the presence of absorption band in around $940 \mathrm{~nm}$. A closer look at this band is shown in the inset of Fig. 4. It can be seen that the intensity of this band increases with increasing concentration of the CuS nanoparticles. This can be attributed to LSPR band of CuS nanoparticles in PVA matrix. It can be seen that there is a red shift in the position of LSPR band of $\mathrm{CuS}$ nanoparticles when they are incorporated in PVA. As the LSPR bands are affected by size, shape and the environment or the matrix of the nanoparticles, hence, a shift in the position in this case can be attributed to the PVA matrix. The LSPR band around $940 \mathrm{~nm}$ is considered good for the photo thermal treat- 
ment (PTT) [17]. Thus, CuS doped PVA films may be explored as possible future material for PTT.

\section{Determination of Absorption Edge and Optical Band Gap}

The absorption coefficient $\alpha(\alpha=A / d)$ where $A$ is absorbance and $d$ is the thickness of the film) as a function of energy of pristine PVA and PVA/CuS films are displays in Fig. 5. The value of absorption edge is determined from the extrapolation of the linear portion of the curve to a point $\alpha\left(\mathrm{cm}^{-1}\right)=0$. The absorption edge for pristine PVA is found to be $5.50 \mathrm{eV}$ which gradually decreases in case of doped films and is found to be $4.65 \mathrm{eV}$ for PVA-CuS-10 \% film indicating a red shift of the absorption edge due to doping $\mathrm{CuS}$ nanoparticles. The value of absorption edge for pure PVA and PVA$\mathrm{CuS}$ films are listed in Table 1.

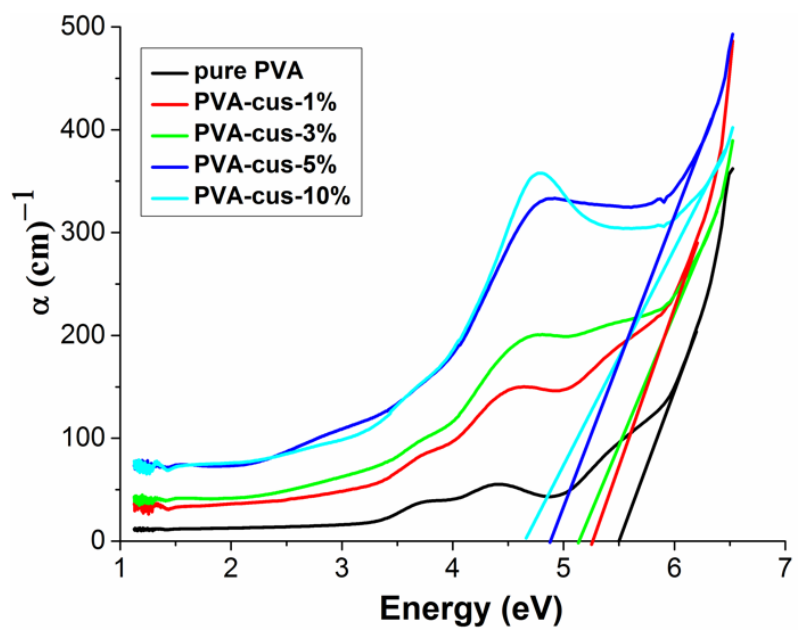

Fig. 5 - Plot of absorption coefficient vs. Energy of pure PVA and PVA-CuS films

The optical band gaps $\left(E_{g}\right)$, is a very important parameter for the polymer samples and is an essential design parameter for many devices like organic solar cells and optoelectronic devices. The optical absorption spectrum is an important tool for determining the optical band gap of materials, crystalline as well as amorphous. Davis and Mott reported the following relation which gives the absorption coefficient dependence on photon energy [18].

$$
(\alpha h v)=B\left(h v-E_{g}\right)^{m},
$$

where $B$ is a constant, $\alpha$ is absorption coefficient of the material, $h v$ is the photon energy and $E_{g}$ is the band gap, $m=2$ for indirect allowed transitions and $m=1 / 2$ for direct allowed transitions. The plots of $(\alpha h v)^{1 / 2}$ versus the photon energy are plotted in Fig. 6. Extrapolation of the linear portion of the curve to a point $(\alpha h v)^{1 / 2}=0$ gives the indirect band gap (IDBG). PVA can have both direct and indirect band gaps. The value of indirect band gap for pristine PVA is $5.05 \mathrm{eV}$ and for the PVA-CuS films, it ranges $4.76 \mathrm{eV}$ to $3.76 \mathrm{eV}$ ( 1 to 10 wt. \% $\mathrm{CuS}$ in PVA). Similarly, plot of $(\alpha h v)^{2}$ versus the photon energy had been used for obtaining the direct band gap (DBG) for pure PVA and PVA-CuS films as shown in Fig. 7. The direct band gap for pure PVA is found to be $5.62 \mathrm{eV}$ and
5.60-5.22 eV for PVA-CuS films (1 to 10 wt. \% $\mathrm{CuS}$ in PVA) as obtained from the extrapolation of the linear portion of the curve to a point $(\alpha h v)^{2}=0$. The indirect and direct band gaps of PVA are found to decrease with increasing doping concentration of $\mathrm{CuS}$ nanoparticles into the PVA film. The results are presented in Table 1. The reduction in band gap may be attributed to the creation of localized energy states due to doping of $\mathrm{CuS}$ nanoparticles.

The indirect and direct band gaps of PVA as well as PVA-CuS films as a function of doping concentration of $\mathrm{CuS}$ is plotted in Fig.8. One can see that reduction of

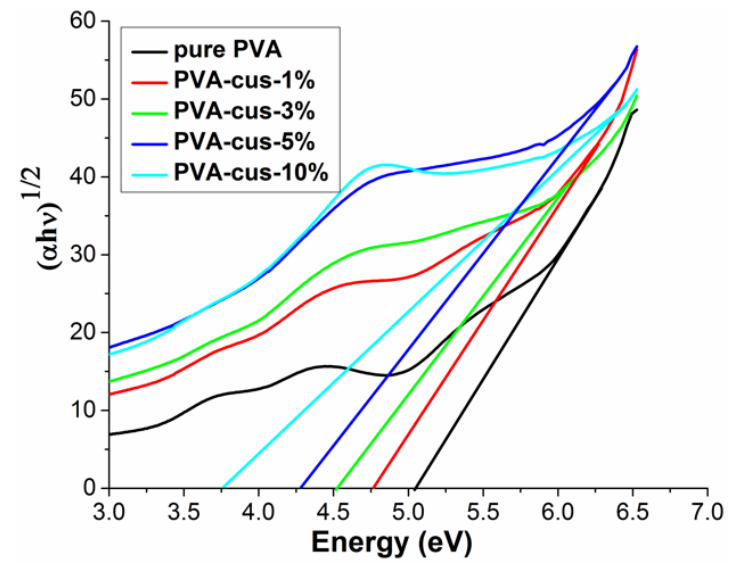

Fig. 6 - Plot of $(\alpha h v)^{1 / 2}$ vs. Energy of pure PVA and PVA-CuS films

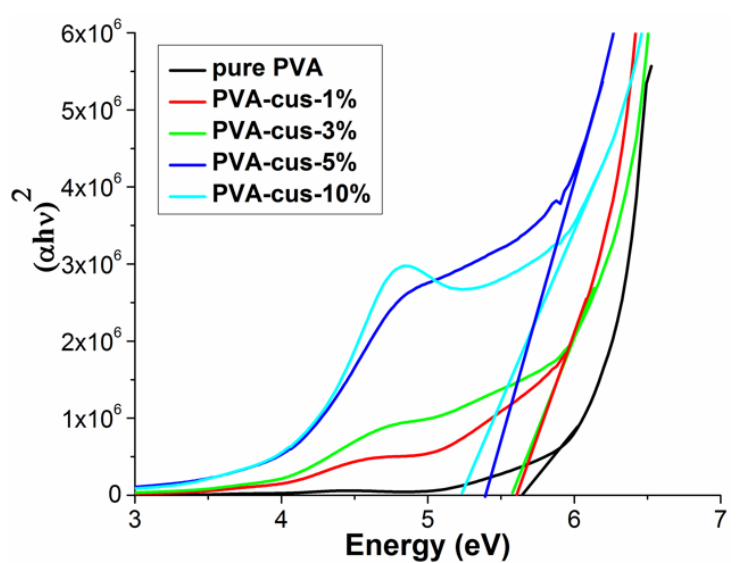

Fig. 7 - Plot of $(\alpha h v)^{2}$ vs. Energy of pure PVA and PVA-CuS films

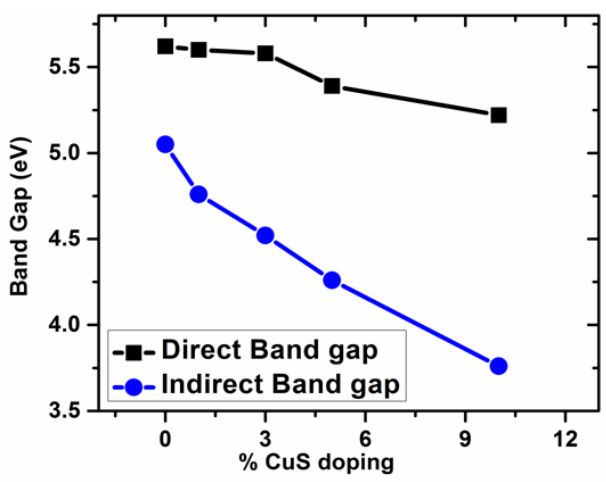

Fig. 8 - Variation of the optical band gap of PVA films with $\mathrm{CuS}$ nanoparticles 
Table 1 - Absorption bands, Absorption edge, Indirect band gap, Direct band gap, refractive index and metallization criterion of films

\begin{tabular}{|l|l|l|l|l|l|l|}
\hline Samples & $\begin{array}{l}\text { Abs. } \\
\text { band } \\
(\mathrm{nm})\end{array}$ & $\begin{array}{l}\text { Abs. } \\
\text { Edge } \\
(\mathrm{eV})\end{array}$ & $\begin{array}{l}E_{\text {gd }} \\
(\mathrm{eV})\end{array}$ & $E_{\text {gi }}(\mathrm{eV})$ & $n_{\text {Dim. }}^{\text {Egi }}$ & $M^{(\text {Egi })}$ \\
\hline Pure PVA & $\begin{array}{l}280 \\
330\end{array}$ & 5.50 & 5.62 & 5.05 & 1.99 & 0.50 \\
\hline $\begin{array}{l}\text { PVA-CuS- } \\
1 \%\end{array}$ & $\begin{array}{l}273 \\
330\end{array}$ & 5.26 & 5.60 & 4.76 & 2.04 & 0.49 \\
\hline $\begin{array}{l}\text { PVA-CuS- } \\
3 \%\end{array}$ & $\begin{array}{l}262 \\
330\end{array}$ & 5.14 & 5.58 & 4.52 & 2.08 & 0.48 \\
\hline $\begin{array}{l}\text { PVA-CuS- } \\
5 \%\end{array}$ & 259 & 4.87 & 5.39 & 4.26 & 2.12 & 0.46 \\
\hline $\begin{array}{l}\text { PVA-CuS- } \\
10 \%\end{array}$ & 257 & 4.65 & 5.22 & 3.76 & 2.22 & 0.43 \\
\hline
\end{tabular}

indirect band gap is more pronounced as compared to direct band gap.

According to Dimitrov and Komatsu good relationship holds between the optical band gap (Eg) and highfrequency refractive index [19]. Using this relationship as shown in equation (2) the high frequency refractive index of pure PVA and PVA-CuS system can be determined by using band gap.

$$
\frac{n^{2}-1}{n^{2}+2}=1-\sqrt{\frac{E_{g}}{20}}
$$

The refractive indices of various films obtained by above mentioned formula are listed in Table 1. Refractive index is showing an increasing trend with increasing doping concentration of CuS nanoparticles.

The Metallization criterion is a factor which is sometimes used to understand the behavior of a material. The theoretical value of Metallization criterion can be evaluated using band gap [19, 20] through the following relation

\section{REFERENCES}

1. P. Ghosh, G.S. Mukherjee, Polym. Adv. Technol. 10, 687 (1999).

2. M. Banerjee, A. Jain, G.S. Mukherjee, Polym. Compos. 40, E765 (2019).

3. M. Banerjee, A. Jain, G.S. Mukherjee, Def. Sci. J. 68, 225 (2018).

4. F. Yakuphanoglu, G. Barım, I. Erol, Physica B 391, 136 (2007).

5. Peng-fei Xue, Ji-bin Wang, Yu-bin Bao, Qiu-ying Lia, Chifei Wu, Chin. J. Polym. Sci. 30, 652 (2012).

6. R.F. Bhajantri, V. Ravindrachary, A. Harisha, V. Crasta, S.P. Nayak, B. Poojary, Polymer 47, 3591 (2006).

7. H.M. Zidan, J. Appl. Polym. Sci. 88, 104 (2003).

8. M. Banerjee, P. Sachdev, G.S. Mukherjee, J. Appl. Phys. 111, 094302 (2012).

9. P. Sachdev, M. Banerjee, G.S. Mukherjee, Def. Sci. J. 64, 290 (2014).

10. A. Joshi, G.S. Mukherjee, M. Banerjee, M. Gupta, A. Choudhary, AIP Conf. Proc. 2100, 020176 (2019).

$$
M=\sqrt{\frac{E_{g}}{20}}
$$

The materials which possess metallization criterion (M) value close to 1 are generally considered to be insultors [18]. The obtained values of metallization criterion of the pristine PVA as evaluated from indirect band gap is found to be 0.50 . The metallization criterion of pristine PVA, as well as PVA-CuS films is presented in Table 1 . The values of metallization parameter implycates that the doped samples tend to be a bit more optically conductive (i.e., less insulating) as a result of the more compact structure of the polymer chains which corroborates with the trend of decreasing band gap values (Table-1); illustratively band gap (Egd, Egi) of PVA and PVA-CuS- $10 \%$ is $(5.62,5.05 \mathrm{eV})$ and $(5.22, \quad 3.76 \mathrm{eV})$ vis-à-vis their metallization factors 0.50 vis-à-vis 0.43 respectively. Interestingly, such trends of metallization criterion are in conformity with the expected trend of change in the band gap of the film with the increase in the doping.

\section{CONCLUSIONS}

Free-standing films of poly vinyl alcohol (PVA) and PVA-CuS films can be successfully prepared. The optical properties like band gap, absorption coefficient, refractive index and metallization criterion of pristine as well as PVA-CuS films have been evaluated. It can be seen that CuS nanoparticles doping can affect the optical properties of PVA. The indirect band gap reduces appreciably on incorporating CuS nanoparticles in the film and refractive index of doped films has high value up to 2.22 hence it can be used as important candidate material for the development of opto-electronics devices, and some organic solar cells.

\section{AKNOWLEDGEMENTS}

The authors would like to thank UGC-DAE-CSR, Indore especially Dr. Mukul Gupta for recording XRD.

11. A. Joshi, G.S. Mukherjee, M. Banerjee, M. Gupta, AIP Conf. Proc. 2220, 020073 (2020).

12. T.S. Soliman, A.S. Abouhaswa, J. Mater. Sci. Mater. Electron. 31, 9666 (2020).

13. D. Singh, S. Kumar, R. Thangaraj, Phase Transitions 87 No 2, 206 (2014).

14. M. Banerjee, P. Sachdev, G.S. Mukherjee, J. Sci. Conf. Proc. 1, 86 (2009).

15. S. Mahendia, A.K. Tomar, R.P. Chahal, P. Goyal, S. Kumar, J. Phys. D. Appl. Phys. 44, 205105 (2011).

16. L.S. Chongad, A. Sharma, M. Banerjee, A. Jain, J. Phys. Conf. Ser. 755, 012032 (2016).

17. Xijian Liu, Bo Li, Fanfan Fu, Kaibing Xu, Rujia Zou, Qian Wang Bingjie Zhang, Zhigang Chena, Jun Quing Hu, Dalton Trans. 43, 11709 (2014).

18. E.A. Davis, N.F. Mott, Philos. Mag. 22, 903 (1970).

19. V. Dimitrov, T. Komatsu, J. Univ. Chem. Technol. Metall. 45 No 3, 219 (2010).

20. R. Indrakanti, V. Brahmaji Rao, C.U. Kiran, J. Mater Sci: Mater Electron. 31, 3238 (2020). 